\title{
Current Mask Generation: an Analog Circuit to Thwart DPA Attacks
}

\author{
Daniel Mesquita ${ }^{1}$, Jean-Denis Techer ${ }^{1}$, Lionel Torres ${ }^{1}$, Michel Robert ${ }^{1}$, \\ Guy Cathebras ${ }^{1}$, Gilles Sassatelli ${ }^{1}$, Fernando Moraes ${ }^{2}$ \\ ${ }^{1}$ LIRMM - Université Montpellier II - France \\ \{mesquita, techer, torres, robert, cathebras, sassate\}@lirmm.fr \\ ${ }^{2}$ PUCRS - Porto Alegre - Brazil \\ moraes@inf.pucrs.br
}

\begin{abstract}
This work addresses the leakage information problem concerning cryptographic circuits. Physical implementations of cryptographic algorithms may let escape some side channel information, like electromagnetic emanations, temperature, computing time, and power consumption. With this information, an attacker can retrieve the data that is being computed, like cryptographic keys. This paper proposes a novel method to thwart DPA attacks, based on power consumption control. As main contribution, this approach not requires any modification on the cryptographic algorithm, the messages or keys.
\end{abstract}

\section{Introduction}

The main objective of cryptographic systems is to allow the communication between two agents, among an insecure channel, with privacy. To accomplish this task, modern cryptographic algorithms uses complex mathematical functions and large keys. In this context, "large" means a number sequence with a range between 128 and 4096 bits.

Cryptographic algorithms are commonly classified in two categories: symmetric and asymmetric. The symmetric ones use the same key to encrypt and to decrypt messages. That supposes a secure channel to accomplish the key exchange, but secret key based algorithms are very performing. On the other hand, asymmetric crypto algorithms uses a pair of keys, mathematically dependent, where one key remains secret, and the other must be published. This kind of algorithms can be used

\footnotetext{
- This work has been partially supported by the Brazilian agency CAPES (Project No 0276-02/2)
} 
to perform digital signatures and authentication schemes. However, public key algorithms are less performing that secret key ones.

Actually, the two classes of algorithms are commonly combined. With a public key algorithm a secure channel can be established. First of all, the users have they origin ascertained with the authentication protocol. Then, they can exchange the symmetric algorithm's secret key, by encrypting it with the asymmetric algorithm. So, the users can communicate on a secure channel.

This idea can be applied to a cellular-to-cellular communication, to a web based video conference, and many other context. Among these, a very growing trend concerns embedded crypto system, like smartcards to ID or credit cards. For instance, in France, each credit card has a memory and a crypto processor. This secure device runs the RSA [1] (asymmetric) and the 3-DES ${ }^{2}$ [2] (symmetric) algorithms. Nowadays there is 45 million of this kind of credit cards, and in the next years, secure smartcards can become a European standard [3].

RSA and AES are crypto algorithms that are proven as being mathematically robust under some conditions. However, the weaknesses of such algorithms are frequently based on implementation problems. Factors like bad random number generation and others can compromise the whole system security. Concerning hardware implementations, even a careful designer cannot avoid a specific class of cryptanalysis.

The hardware devices implementing cryptographic algorithms (processors, ASIC, FPGA and others), may leak some information, like electromagnetic emanations, computing time and power consumption. By analyzing one or more of these information, an attacker can relate the leaked data with the device's internal state, and so, with the secret key. This kind of attack is called Side Channel Attack (SCA).

The SCA most famous is the Differential Power Analysis (DPA) [4]. The DPA attack is very efficient and relatively low cost. Power analysis principle is based on the current consumption to compute logical 0 (zeros) and logical 1 (ones), that is different for each case. Differential Power Analysis enables an intruder to extract secret keys and information from smartcards, which can be used to create fraudulent transactions, generate counterfeit digital cash or perform content piracy. DPA eavesdrops on the fluctuating electrical power consumption of the microprocessors at the heart of these devices, and uses advanced statistical methods to extract cryptographic keys and other secrets. Although DPA attacks currently require a high level of technical skill in several fields to implement, they can be repeated using a few thousand dollars worth of standard equipment, and can often break a device in a few minutes.

After a while, some efficient algorithmic countermeasures have been presented, but most of them rely on the modification at the algorithm level, to avoid the correlation between the power consumption, the message and key data. Our original approach simplifies this task by masking power consumption, without any algorithmic modification.

This paper is organized as follows: Section 2 describes the DPA attack. Section 3 shows previous and related works on DPA countermeasures. Section 4 presents the new method to avoid DPA attacks, and conclusions are discussed and future works shown in Section 5.

\footnotetext{
${ }^{2}$ In the next months, all credit cards will be substituted by others running the AES algorithm
} 


\section{DPA Attack}

DPA attacks use statistical techniques to determine secret keys from complex, noisy power consumption measurements [4]. For a typical attack, an adversary repeatedly samples the target device's power consumption through each of several thousand cryptographic computations with the same key. These power traces can be collected using high-speed analogical-to-digital converters, using digital storage oscilloscopes. Figure 1 illustrates this method.

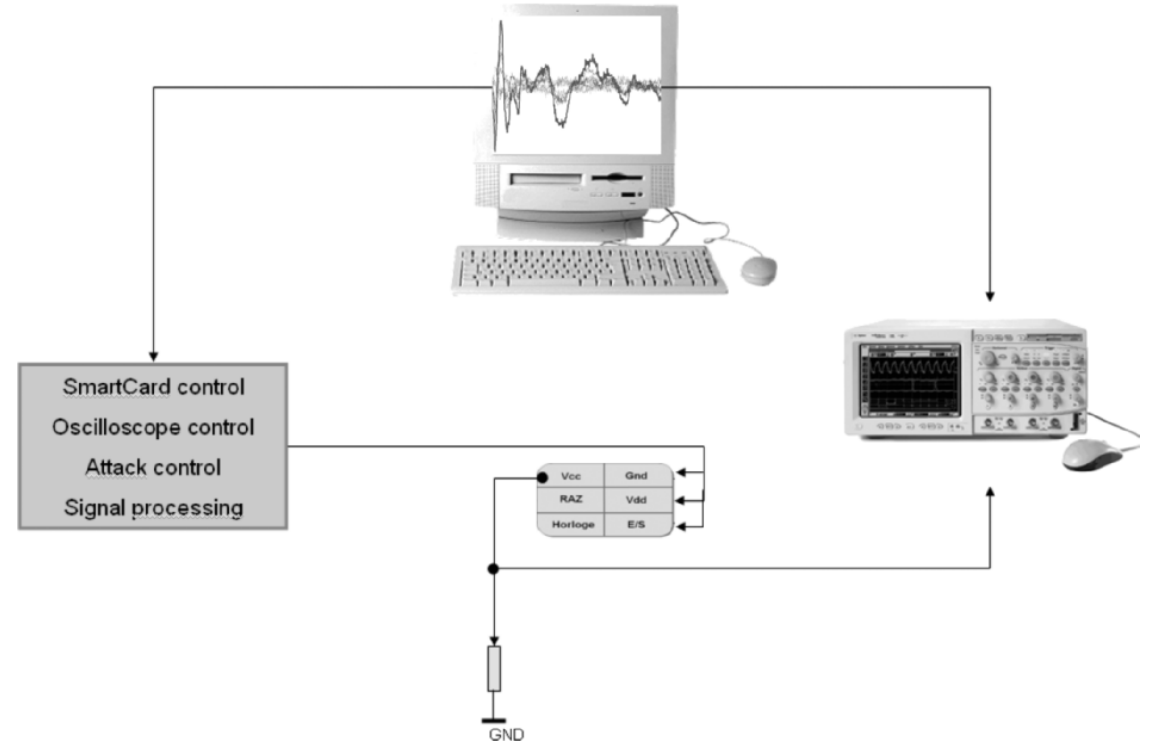

Figure 1 - A DPA attack platform

Because it's widespread use, the DES algorithm is used to explain a DPA attack. DES executes in 16 steps, called rounds. In each round, a transformation $F$ is performed on 32 bits. This $\mathrm{F}$ function uses eight non-linear transformations from 6 bits to 4 bits. Each of such transformations is called S-Box. Figure 2 show the DES scheme. Initially the algorithm receives the key and performs a key division into sub keys (a). Then the plain text is transformed trough permutations and substitutions with the sub keys. The DES is composed by 16 rounds of substitutions (b), where the most important elements are the substitution boxes (S-Box). The DPA attack is performed targeting only one S-Box.

First, it is needed to make some measures (1000 samples, for instance) from the first (or the last) round of DES computation. After that the 1000 curves are stocked, and an average curve $(A C)$ is calculated.

Secondly, the first output bit $(b)$ of the attacked S-box is observed. This $b$ bit depends only of the 6 bits from the secret key. Then, the attacker can make an hypothesis on the involved bits. He computes the expected values for $b$; this enables to separate the 1000 inputs into two categories: those giving $b=0$ and those giving $b=1$. 
Thirdly, the attacker computes the average curve $A C^{\prime}$ corresponding to inputs of the first category. If $A C^{\prime}$ and $A C$ have a difference much greater than the standard deviation of the measured noise), it means that the chosen values for the 6 key bits are correct. But, if $A C^{\prime}$ and $A C$ do not show any visible difference, the second step must be repeated with another hypothesis for the 6 key bits.

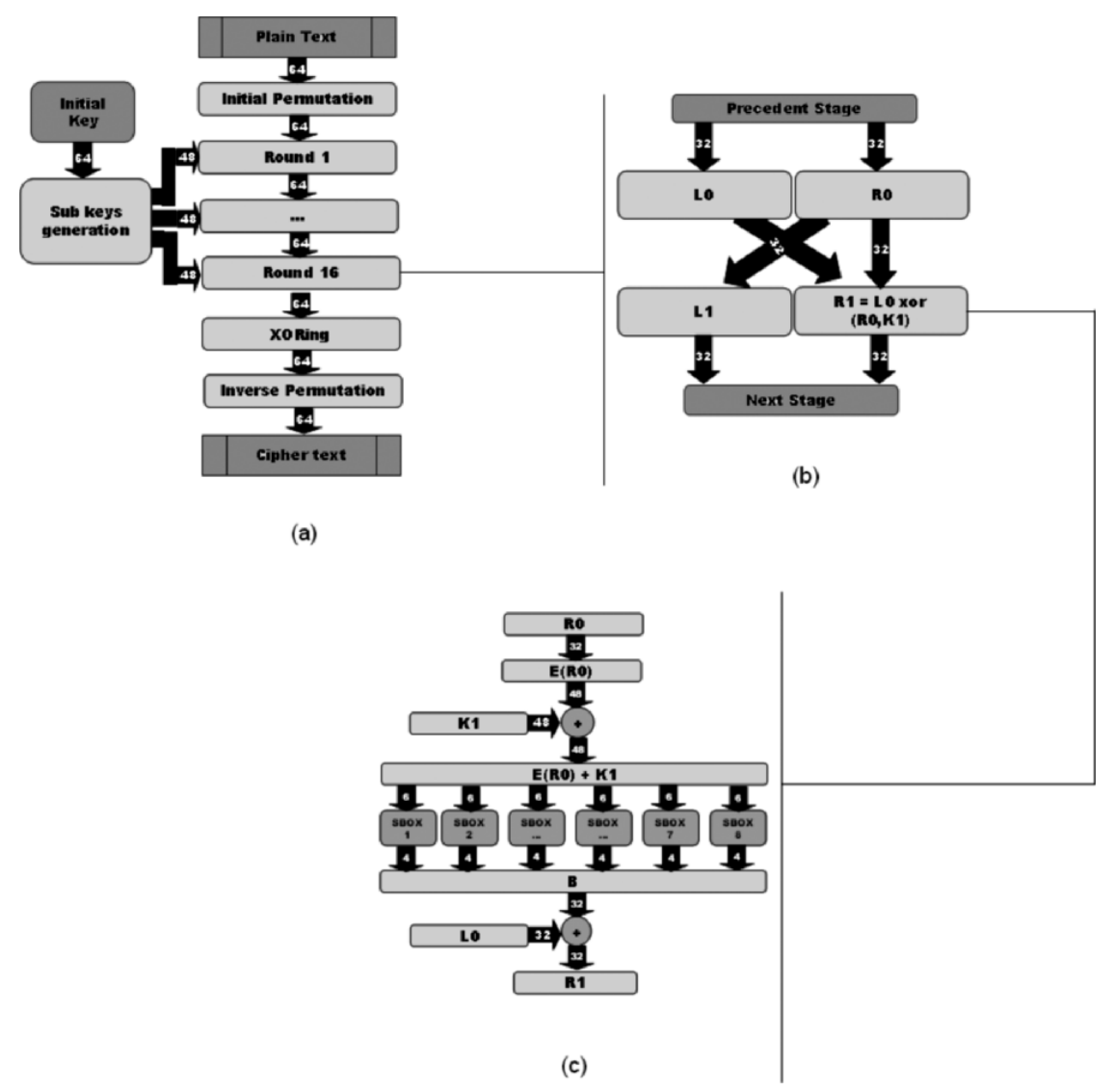

Figure 2 - The DES Algorithm (a), with the round details (b), and emphasizing the importance of the S-Boxes (c).

Afterwards, the second and third steps must be repeated with a target bit $\mathrm{b}$ in the second S-box, then in the third, and so on, until the eight S-Box. As a result, the attacker can obtain the 48 bits of the secret key. Finally, the remaining 8 bits can be retrieved by exhaustive search.

More details of DPA attacks against DES can be found in the reference [6]. 


\section{Related works}

The countermeasures that have been developed against DPA attacks until now can be classified in two families. The first group is composed by the algorithmic countermeasures. The basic idea from references [5], [6], [7] and [8] is to randomize the intermediate results that are produced during the computation of a cryptographic algorithm. Classical DPA attacks can be impracticable if these countermeasures are well implemented. But these randomizations are quite expensive to implement for non-linear operations as they are used in algorithms like DES and AES. Furthermore, the algorithmic approach does not provide sufficient protection against high-order DPA attacks [19]. As consequence, this kind of method needs complementary hardware countermeasures. The next subsections shows some algorithmic and hardware countermeasures.

\subsection{Algorithmic Countermeasures}

There are several algorithmic (or software) countermeasures to thwart DPA attacks. Some of the first ones were proposed in [20], and the three proposed countermeasures are efficient against SPA and classical DPA attacks. For RSA cryptosystems the first method described by Coron is applicable, and the second one is just an adaptation of the Chaum's blind signature [21]. The third method is only suitable for ECC (Elliptic Curve Cryptosystems). But the recently proposed Refined Power Analysis (RPA) [22] overrules these countermeasures.

The BRIP method counteracts the RPA but is also targeted to ECC, not tailored to work with the widely used RSA algorithm [23]. The message blinding proposed by P. Kocher [24] seems to be an efficient countermeasure against the MRED [25] (an attack targeting CRT implementation of RSA).

In general, the countermeasures protecting the RSA algorithm of DPA attacks relies on message or exponent blinding. These methods contribute or not to the security of the system, depending on the way they are implemented and the kind of attack. Is not rare that defense against one attack may benefit another type of attack.

So, the best way to counteract DPA attacks is to target the DPA principle: the correlation between the data computed and the power consumption. Differently of the works that generally proposes CRT to accelerate RSA, like [26], another approach proposes a full RNS representation to compute RSA [27], [28]. Besides the acceleration, a full RNS implementation of RSA can intelligently be used to counteract DPA and DFA attacks, by altering the intermediate data through an exotic arithmetic. The problem with these approaches is that they require a full changing on the cryptographic algorithm to adapt it to the new arithmetic.

\subsection{Hardware Countermeasures}

The hardware method to counteract DPA attacks differs expressively from the algorithmic one. For the hardware approach the intermediate results of the cryptographic algorithm computation are not affected. As an alternative, the contribution of the hardware approach is to hide the attackable part of the power 
consumption with different noises. The noise addition has a direct relation with the needs of measurement. It does not avoid DPA attacks, but makes it quite more difficult. The effectiveness of the countermeasures against DPA is due to the fact that cryptographic devices are typically protected by a combination of algorithmic and hardware techniques, or only the hardware one [9].

In order to decrease the correlation between data inputs and the power consumption of a given circuit, we must be able to increase the samples needed in DPA. Two major hardware countermeasures in this sense have been proposed. The first one concerns the reduction of the signal-to-noise ratio (SNR). For definition of SNR we call $I_{c}$ the current consumption of the attacked circuit at a given moment $\mathrm{t}$. In is the current noise caused by the hardware countermeasure. So, the current consumption can be written as $I_{\text {total }}=I_{c}+I_{n}$. The k variable is the signal attenuation caused by the $I_{n}$ current. The SNR definition is given by Equation (1).

$$
S N R=20 \times \log \left(\frac{I_{c}}{k R}\right)
$$

Lower SNR is lower the correlation between the correct hypothetical current consumption and the real power consumption of the device. To reduce SNR there are some works that use special logic to minimize the data dependency of the current consumption.

In the references [10] and [11] the balanced dual-rail logic is proposed. The basic idea is that a logic gate must consume an equivalent power, independently from the incoming input values. The SNR is reduced by this data-independent switching of the standard cells. Unfortunately, the experiments show that this goal is only partially reached. Dual-rail approach is not sufficient to guarantee a complete data independent power signature. One potential problem is that the gate loads may differ due to differences in routing. The design of each dual-rail gate must ensure equal input pin loads and balanced power usage. To achieve this, the process of grouping cells in the placement must be done carefully, which implies a high development effort. Besides that, the final circuit with dual-rail logic takes about tree times the area and two times the consumption of the original circuit.

The second hardware approach to prevent DPA attacks is to reduce the correlation between input data and power consumption by randomly disarrange the moment of time at which the attacked intermediate result is computed. If the time tc is different in every power trace, the correlation between the hypothetical power consumption and the real one is highly reduced. The countermeasure proposed by [12] lies on the insertion of random delays. The method described in [9] counteracts the DPA by using power-managed blocks to mask the power consumption. Both approaches [13] and [14] increase the difficulty the DPA attack. But, as shown in [15], even if a direct calculation of the maximum probability of a given power consumption occurring at a given time is not practical, it is always possible to approximate it empirically based on a software model of the countermeasure.

This work gives a trend to mask the power consumption not by randomizing the consumption or creating noise but by generating, at the transistor level, a constant consumption. It is a little similar with the work proposed by Adi Shamir in [17], concerning the approach's level of abstraction. But the circuit described in [17] considers only if the attacker probes the $V c c$, because the Gnd line remains vulnerable. Also, the two capacitors proposed are too big to be integrated (100nF) or 
a System in Package approach should be considered [18]. As explained in next session, our circuit masks the consumption even if the attack occurs in the Vcc or in the Gnd lines.

\section{Current Mask Generation technique}

Based on the decreasing Signal to Noise Ratio idea, we conceived an analogical circuit able to mask the real power consumption from a cryptographic circuit. The main goal of this approach is to increase the security of the crypto devices without any modification of the cryptographic algorithm implemented. In addition, no special standard cells are required, unlike the dual-rail approach.

Our technique tries to mask the power consumption by normalizing the current consumed by the cryptographic circuit $(C C)$. This task is accomplished by an analogical circuit called Current Mask Generator $(C M G)$, which's role is to maintain the total current constant (from an external view). To design the $C M G$, firstly some measures were made, in order to establish the CC's peak of current consumption. Once this value detected, the objective is to remain at this peak, even if the CC's consumption is lower than it.

The CMG is composed basically of a high-swing current mirror, a follower circuit, and a small capacitance. As can be depicted from Figure 2, the CMG acts aside of the $\mathrm{CC}$, this outlines that any change in the cryptographic circuit is required. Note that the CMG and the cryptographic circuit are not in the same scale. Actually, the CMG takes only $30 \%$ of a standard hardware implementation of the DES algorithm.

Still in Figure 2, the current mirror acts imposing a fixed current $\left(I_{2}\right)$. I2 is given by the w coefficients from P1 and P0, as can be viewed in Equation (2) and is equals to the CC's peak of current consumption.

$$
I_{2}=\left(\frac{w P_{1}}{w P_{0}}\right)
$$

The cryptographic circuit consumes a $I_{c}$ current. When $I_{c}=I_{2}$, it means that the $C C$ consumes all current furnished, and the $C M G$ must stand by. Otherwise, when the $C C$ not requires all $I_{2}$ current, then the circuit follower performs a feedback-loop to consume a current $I_{L}$ so that $I_{L}=I_{2}-I_{c}$.

In fact, the circuit follower plays as a voltage generator. The operational amplifier receives a tension from the mirror and compares it with a reference voltage (i.e. $\left.V_{e x t}\right)$. ). If the cryptographic circuit consumes an amount of current less than $I_{2}$, the voltage at the operational amplifier (Op-Amp) input will be lower than the reference voltage. Then the output of the Op-Amp will send $O$ to the $P_{4}$ transistor. So, it will consume an $I_{L}$ current, that is the difference between $I_{2}$ and $I_{c}$. When the $C C$ consumes at the peak (i.e. $I_{c}=I_{2}$ ), the Op-Amp sends a 1 to the $P_{4}$, switching off the transistor, because it is no longer necessary to drain current.

Finally, the 9,5pF capacitor's function is to give some time to the feedback-loop react. Also, the capacitor smoothes the tension, what have a benefice effect to the consumption masking. 


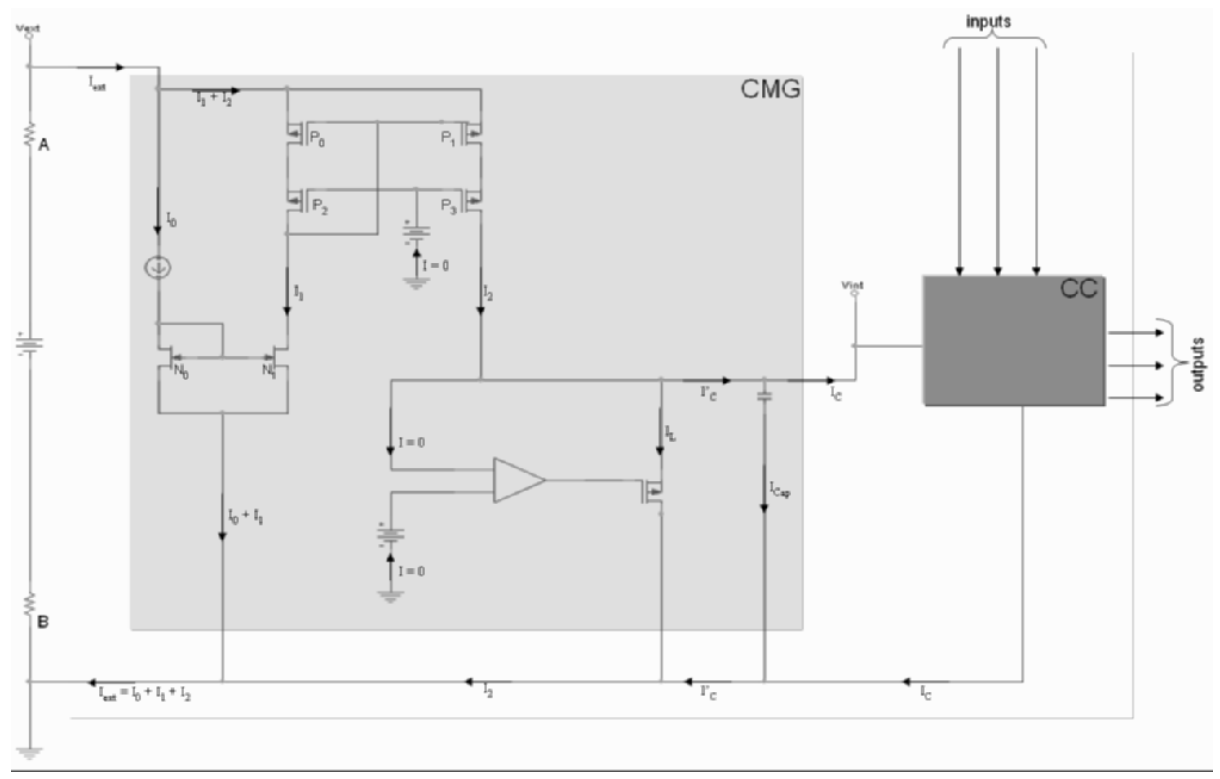

\section{Figure 3 - The CMG circuit in detail}

To validate the $C M G$ method, it is used a DES S-box, to play the role of $C C$. Then, the S-box was simulated to determine the current consumption worst case. The Figure 4 shows a consumption peak about $6 \mathrm{~mA}$.

Many simulations were made for different data scenarios. As can be viewed in Figure 5, the $C M G$ works efficiently, masking the $C C$ current consumption, and making DPA attacks a very difficult task. The signal /R8/Plus is the current consumed by the CC and the signal /R4/Plus is the masked signal.

The Figure 6 shows, from a top-down view, the current consumption that can be plotted from the external $V d d$ or $G n d$, the current consumption of the cryptographic circuit, de data input called $a l$ and the data input called $a 0$. Analyzing the consumption reported to data input, Figure 6 shows that even with a one or a zero, or two ones, or two zeros as entries, the consumption viewed at the attackers side remains the same.

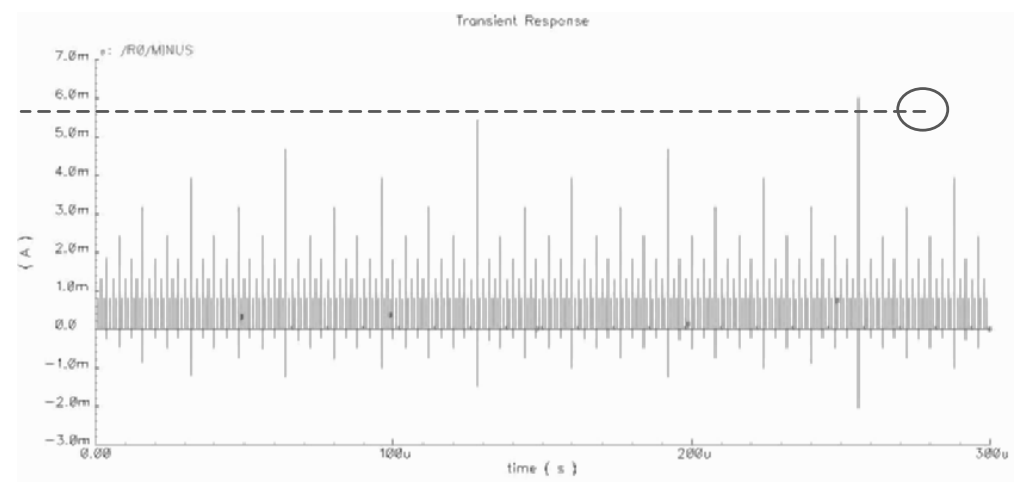

Figure 4 - The S-box current consumption 


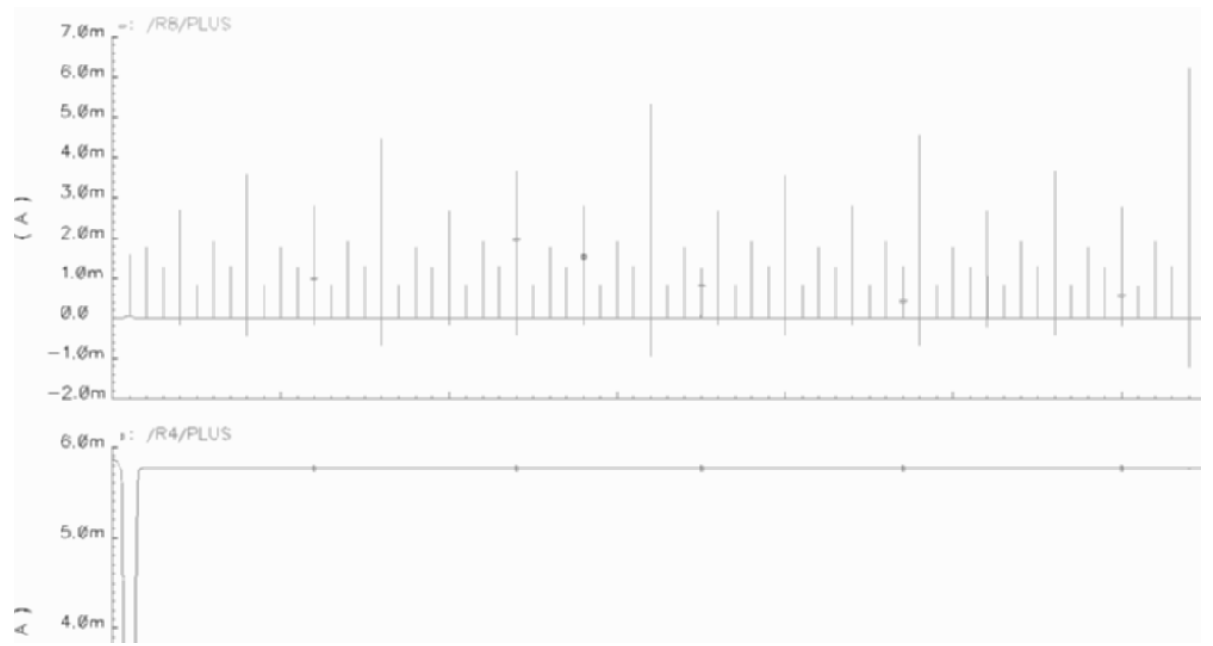

Figure 5 - CC's current consumption (R8 Plus) and the current provided by the CMG circuit (R4 Plus).

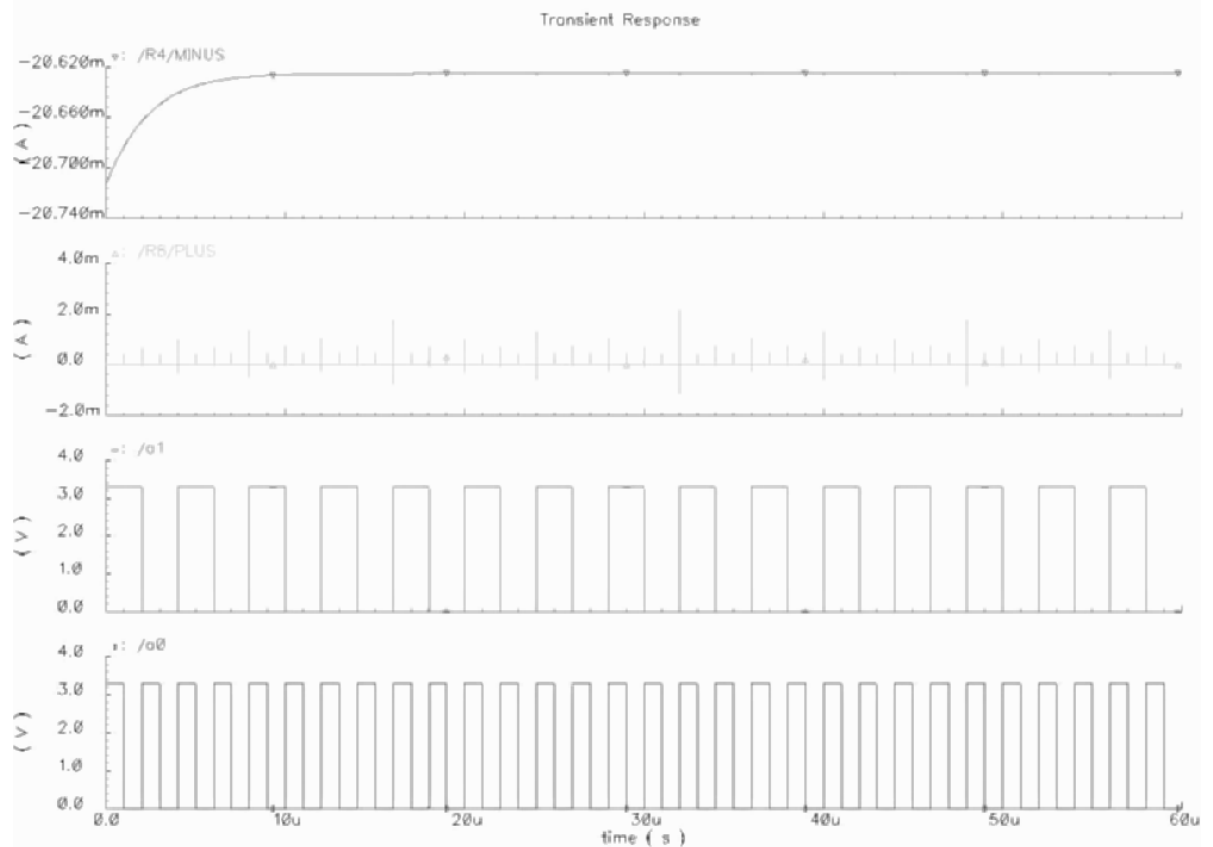

Figure 6 - The current provided by the CMG circuit, the current consumed by the CC, and some data input

To define the difficulty to make a DPA attack, some parameters must be considered. The first one is the Signal to Noise Ratio. Contrary to a normal multimedia application, were the designer search to increase the SNR, by decreasing the noise as much as possible, the CMG approach intends the opposing: decrease the signal. 
Figures 5 and 6 show glitches on the masked signal /R4/Plus. If a zoom is done, the same pattern found in $/ R 8 / P$ lus is repeated in $/ R 4 / P l u s$. It signifies that the system is not perfect. But if the values of each signal are considered (see Figure 7), it is clear that the $\mathrm{CMG}$ attenuates the current by a factor $k \approx 20$.

This $k$ factor is obtained by measuring the $/ R 8 / P l u s$ signal's difference between its peak and its minor value (still in Figure 7), which done a $C C_{\text {delta }}$. Then, the process is repeated for the $/ R 8 /$ Plus signal, obtaining a $C M G_{\text {delta }}$ value. So,

$$
k=\left(\frac{C C_{\text {delta }}}{C M G_{\text {delta }}}\right)
$$

To view the CMG attenuation, the Signal to Noise Ratio show in the Equation (1) must be expanded to Equation (4):

$$
\begin{aligned}
& S N R_{C M G}=20 \times \log \left(\frac{I_{c}}{k \times N}\right)=20 \times \log \left(\frac{I_{c}}{N}\right)-20 \times \log (k) \\
& S N R_{C M G}=S N R-20 \times \log (k)
\end{aligned}
$$

With the equation (4), and regarding Figure 8 for the given example, the current viewed by an attacker is smoothed by $25 \mathrm{db}$. It means that the observed signal could be drowned into the noise (Figure 8 (b)).

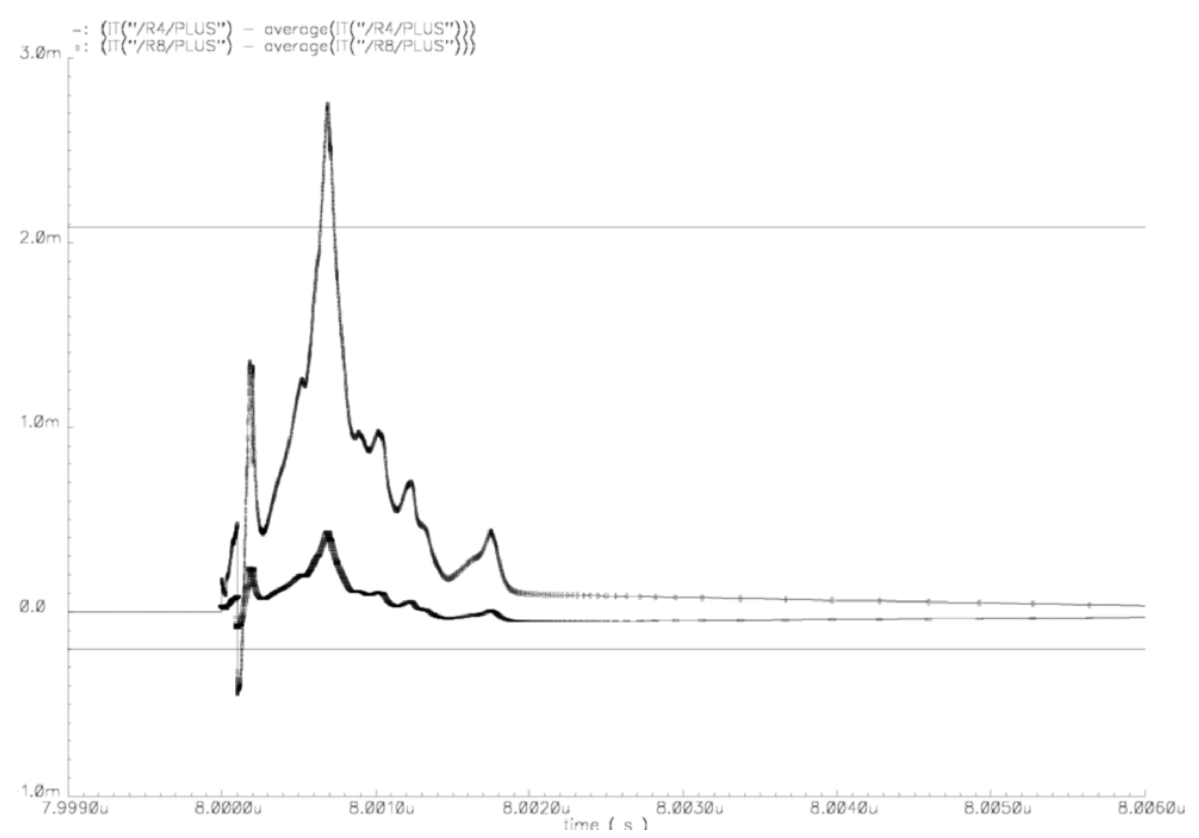

Figure 7 -The signal attenuation reached by the CMG 

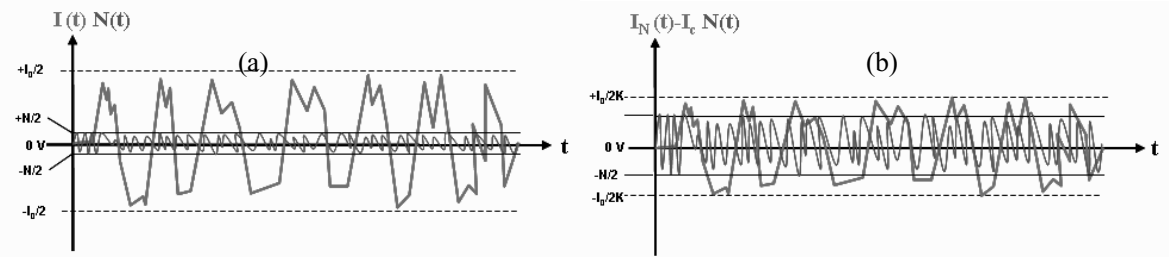

Figure 8 - Normal power consumption and noise (a) and the power consumption with the CMG, immersed into noise (b)

\section{Conclusion}

The presented work improves the robustness of cryptographic circuits against DPA attacks. In this paper we have proposed a low level solution, which has as major contribution the fact that no changes are needed into the cryptographic algorithm.

Our approach is not only simple to implement, but is also cheap regarding the area overhead point of view. A classic DES circuit has about $16 \mathrm{~mm}^{2}$ of surface (synthesis with the AMS 0.35 technology), while the CMG has only $5 \mathrm{~mm}^{2}$, so the area overhead is only about $30 \%$, which is an acceptable cost to increase robustness on cryptographic systems.

Another cost of this approach is the increased power consumption. But it remains interesting for applications like credit cards, set-top boxes, phone cards and others where the low-power for cryptographic applications is less essential. In banking operations, like cash transactions, the cryptographic operation is not used all the time and the whole user operation is not so time-consuming that justifies a low power approach. The most important in this case is the security. The poor Signal to Noise Ratio generated by the CMG circuit makes a DPA attack very difficult.

On the other hand, as can be viewed in the figure 6, the attenuation could be improved. By modifying the feedback-loop and the current generator we may diminish the Signal to Noise Ratio. One approach is the inclusion of an inductor in series with the current mirror. In our last experiments, preliminary results show attenuation greater than the first version of the $\mathrm{CMG}$, and the trend is that the use of this inductor may lead to the attenuation of electromagnetic emissions too. So the CMG could also improve resistance against electromagnetic analysis (EMA) attacks.

\section{References}

1. Rivest, R., Shamir, A., et al. "A Method for Obtaining Digital Signatures and Public-Key Cryptosystems”. ACM Communications, vol 21. pp. 120-126. 1978.

2. "Data Encryption Standard (DES)". Federal Information Processing Standards Publications (FIPS PUBS) $\mathrm{N}^{\mathrm{0}}$ 46-3. http://csrc.nist.gov/publications/fips/ fips46-3/fips46-3.pdf. EUA.October 25, 1999.

3. Groupement des Cartes Bancaires CB. "Les cartes Bancaires en Nombres 2004". http://www.cartes bancaires.com/FR/info/communiques/2005/DPchiffresCB2004. pdf. Paris, march 2005. 
4. Kocher, P., Jaffe J., et al. “ Differential Power Analysis : Leaking Secrets ”. Advances in Cryptology: Proceedings of CRYPTO'99, Vol. 1666, SpringerVerlag, pp. 388-397. 1999.

5. Messerges, T. S., Dabbish E. A., et al. "Power Analysis of Modular Exponentiation in Smartcards ". Cryptographic Hardware and Embedded Systems CHES 199. Lecture Notes in Computer Science, Vol. 1717, Springer, ISBN: 3-540-66646-X. pp. 144-157, 1999.

6. Goubin, L., Patarin, J. "DES and Differential Power Analysis - The "duplication" method". Cryptographic Hardware and Embedded Systems - CHES 1999. Lecture Notes in Computer Science, Vol. 1717, Springer, ISBN: 3-54066646-X. pp. 158-172, 1999.

7. Trichina, E., De Seta, D. et al. “ Simplified Adaptive Multiplicative Masking for AES ". Cryptographic Hardware and Embedded Systems - CHES 2002. Lecture Notes in Computer Science, Vol. 2523, Springer, ISBN: 3-540-00409-2. pp. 187-197, 2003.

8. Golic, J. D., Tymen, C. "Multiplicative masking and Power Analysis of AES". Cryptographic Hardware and Embedded Systems - CHES 2002. Lecture Notes in Computer Science, Vol. 2523, Springer, ISBN: 3-540-00409-2. pp. 198-212, 2003.

9. Benini, L., Macii, A., et al. "Energy-aware design techniques for differential power analysis protection". Design Automation Conference - DAC 2003. Anaheim, USA. June, 2003.

10. Saputra, H. Vijaykrishnan, N., et al. " Masking behavior of DES encryption ". Design, Automation and Test Europe - DATE 2003. ACM-Sigda, ISBN: 0-76951471-5. Munich, Germany, 2003.

11. Simon M., Ross A., et al. "Balanced Self-Checking Asynchronous Logic for Smart Card Applications", Microprocessors and Microsystems Journal, 27(9). Elsevier, ISSN: 0141-9331. pp. 421-430, October 2003.

12. Clavier, C., Coron, J-S., et al. "Differential Power Analysis in the presence of hardware countermeasures". Cryptographic Hardware and Embedded Systems - CHES 2000. Lecture Notes in Computer Science, Vol. 1965, Springer, ISBN: 3-540-41455-X. pp. 252-263, 2000.

13. Irwin, J., Page D., et al. “ Instruction stream mutation for non-deterministic processors. Internation conference on Application Specific Systems, Architectures and Processors - ASAP 2002. IEEE press. pp. 286-295. 2002 
14. May, D., Muller H. L., et al. "Non-deterministic processors". Information security and privacy - ACISP 2001. Lecture Notes in computer Science, volume 2119. Springer ISBN: 3-540-42300-1. pp. 115-129. Sydney, Australia. July 2001.

15. Mangard, S. "Hardware countermeasures against DPA - a statistical analysis of their effectiveness". Topics in Cryptology - CT-RSA 2004. Lecture Notes in Computer Science, Vol. 2964, Springer, ISBN: ISBN 3-540-20996-4. pp. $222-$ 235. San Francisco, USA. February 2004.

16. Fouque, P.-A., Muller F., et al. "Defeating Countermeasures Based on Randomized BSD Representations". Cryptographic Hardware and Embedded Systems - CHES 2004. Lecture Notes in Computer Science, Vol. 3156, Springer, ISBN: 3-540-22666-4 pp. 312-327. Cambridge, EUA. 2004.

17. Shamir, A. "Protecting smart cards from passive power analysis with detached power supplies". Cryptographic Hardware and Embedded Systems - CHES 2000. Lecture Notes in Computer Science, Vol. 1965, Springer, ISBN: 3-54041455-X. pp.71-77, 2000.

18. Tummala, R. and Madisetti, V. "System on Chip or System on Package?" IEEE Design and Test of Computers Review. Vol. 16, N. 2. IEEE Press. ISSN: 07407475. pp. 48-56, April-June 1999.

19. Kocher, P., Jaffe, J., et al. "Introduction to Differential Power Analysis and Related Attacks". Technical Report, Cryptography Research Inc., 1998. Available from http://www.cryptography.com/dpa/technical/index.html.

20. Coron, J-S. "Resistance against Differential Power Analysis for Elliptic Curve Cryptosystems". Cryptographic Hardware and Embedded Systems, Proceedings of CHES 1999. Lecture Notes in Computer Science, Vol. 1717, Springer-Verlag, ISBN: 3-540-66646-X. pp. 292-302, 1999.

21. Chaum, D. "Security without identification: transaction systems to make Big Brother obsolete". Communication of the ACM. Vol. 8., n 10, pp. 1030-144. 1985.

22. Goubin, L. "A refined power-analysis attack on elliptic curve cryptosystems". Publick Key Cryptography: Proceedings of PKC '03. Lecture Notes in Computer Science, Vol. 2567, Springer-Verlag, pp. 199-210. 2003.

23. Hideyo, M. and Atsuko, M. "Efficient Countermeasures against RPA, DPA, and SPA". Cryptographic Hardware and Embedded Systems, Proceedings of CHES 2004. Lecture Notes in Computer Science, Vol. 3156, Springer-Verlag, ISBN: 3-540-22666-4. pp. 343-356, 2004.

24. Kocher, P. "Timing Attacks on Implementations of Diffie-Hellman, RSA, DSS, and Other Systems". $16^{\text {th }}$ Workshop in Cryptology: Proceedings of Crypto '96. Lecture Notes in Computer Science, Vol. 1109, Springer-Verlag, ISBN: 3-54061512-1, pp. 104-113. Santa Barbara, USA. 1996. 
25. Boer, B. "A DPA Attack against the Modular Reduction within a CRT Implementation of RSA". Cryptographic Hardware and Embedded Systems, Proceedings of CHES 2002. Lecture Notes in Computer Science, Vol. 2523, Springer-Verlag, ISBN: 3-540-00409-2, pp. 228-243, 2002.

26. Kim, C., Ha, J., et al. "A CRT-Based RSA Countermeasure against Physical Cryptanalysis". International Conference on High Performance Computing and Communications: Proceedings of HPCC '05. pp. 549-554, Naples, Italy, 2005.

27. Bajard, J-C., Imbert, L., et al. "A Full RNS Implementation of RSA". IEEE Transactions on Computers. Vol. 53, n 6, pp. 769-774. 2004.

28. Ciet, M., Neve, M., et al. "Parallel FPGA implementation of RSA with residue number systems - can side-channel threats be avoided?". 46 ${ }^{\text {th }}$ IEEE International Midwest Symposium on Circuits and Systems: Proceedings of MWSCAS '03. Cairo, Egypt, December 2003.

29. Bajard, J-C., Imbert, L., et al. "Leak Resistant Arithmetic". Cryptographic Hardware and Embedded Systems, Proceedings of CHES 2004. Lecture Notes in Computer Science, Vol. 3156, Springer-Verlag, ISBN: 3-540-22666-4. pp. 62-75, 2004. 